\title{
Candidal balano-posthitis: a study of diagnostic methods
}

\author{
W G Dockerty, C Sonnex
}

\begin{abstract}
Objectives-To compare microscopy with culture for diagnosing candidal balanoposthitis and to document which diagnostic methods are used in genitourinary medicine clinics in Great Britain.

Design-(a) Penile material for microscopy and fungal culture were obtained from men with balano-posthitis. A "plain-slide" method of collecting material for microscopy was compared with a novel "adhesive-tape" method of sampling. (b) Questionnaires were sent to all genitourinary medicine clinics in Great Britain.
\end{abstract}

Setting-The Department of Genitourinary Medicine, Addenbrooke's Hospital, Cambridge, England.

Main outcome measures-The sensitivity and specificity of microscopy using culture as the "gold standard" for diagnosis. Results-Candida was isolated from 35\% of 450 men with balano-posthitis attending the clinic over a three year period. The sensitivity of microscopy compared with culture was $12 \%$ ("plain-slide" method of material collection) and $65 \%$ ("adhesive-tape" method) (p<0.0001). The respective specificities were $95 \%$ and $81 \%$. The positive predictive values for the two methods of material collection were $50 \%$ ("plain-slide" method) and $75 \%$ ("adhesive-tape" method). The respective negative predictive values were $71 \%$ and $72 \%$. $60 \%$ of 250 genitourinary medicine clinics returned questionnaires. $13 \%$ routinely diagnosed candidal balanoposthitis by appearance only and 34\% sometimes relied only on clinical appearance. Culture was used by $78 \%$ and microscopy by $69 \%$ of clinics. Material for microscopy was most commonly collected by using a cotton-wool tipped swab and the Gram stain was the favoured method for microscopy.

Conclusion-Candida is a common cause of balano-posthitis. Diagnosis by microscopy has a low sensitivity and varies with the method used for collecting material. Although up to one third of genitourinary medicine clinics may rely solely on clinical appearance for diagnosis most continue to use microscopy and culture.

(Genitourin Med 1995;71:407-409)

Keywords: balano-posthitis; candida

\section{Introduction}

Candidal balano-posthitis is a well recognised condition, first described by Engman in $1920 .^{1}$ Most patients present with penile irritation associated with mild penile erythema and small, irregular eroded papules. An acute, fulminating, oedamatous balano-posthitis is occasionally seen, most commonly associated with diabetes. ${ }^{2}$ The diagnosis of candidal balano-posthitis is primarily clinical with confirmation by culture. Although the role of microscopy has not been previously assessed in the male, studies of women with vaginal candidiasis have reported a sensitivity of wet mount microscopy of between $19 \%$ and $52 \%$ compared with culture. ${ }^{34}$ The aims of this study were to compare microscopy with culture for diagnosing candidal balano-posthitis, to assess the use of a novel method of material collection for microscopy and to document which diagnostic methods are currently used in genitourinary medicine (GUM) clinics in Great Britain.

\section{Methods}

Sub-preputial and glans swabs for candida culture were performed on 450 men with balano-posthitis attending the Department of Genitourinary Medicine at Addenbrooke's Hospital, Cambridge between 1991 and 1993. Material for culture was obtained by brushing a foam swab $^{5}$ over the glans penis and subpreputial epithelium and then placing the swab into Feinberg-Whittington (with Stenton's modification) culture medium. ${ }^{67}$ Cultures were incubated at $37^{\circ} \mathrm{C}$ for 18 hours at which time a wet-mount was prepared from a sample of the culture medium and examined by phase contrast microscopy for budding yeast cells. Candida species were identified by their characteristic morphological appearance.

Material for microscopy was obtained by pressing a microscope slide firmly against the glans and sub-prepuce. As the quantity of material collected was often scanty, an alternative method was devised and compared with the original "plain-slide" collection method. This involved attaching double-sided clear adhesive tape to one half of a microscope slide and then pressing the slide against the affected epithelium, as described previously. Slides were heat fixed by short exposure to a flame, so as to avoid damaging the adhesive tape, and then Gram stained prior to microscopy. The level of discomfort produced by material collection for microscopy and, in some cases, culture, was recorded on a scale of $1-5$, where 1 
Table 1 Diagnosis by Gram stain and light microscopy compared to culture

\begin{tabular}{llcc}
\hline \multirow{2}{*}{$\begin{array}{l}\text { Method of material } \\
\text { collection for microscopy }\end{array}$} & $\begin{array}{l}\text { Microscopy } \\
\text { result }\end{array}$ & \multicolumn{2}{c}{ Candida culture } \\
\cline { 3 - 4 } & Positive & Negative \\
\hline "Plain slide" & Positive & 16 & 16 \\
& Negative & 121 & 294 \\
"Adhesive tape" & Positive & 15 & 5 \\
& Negative & 8 & 21 \\
\hline
\end{tabular}

$=$ no discomfort and $5=$ moderately painful. To determine how other GUM clinics diagnosed candidal balano-posthitis, a questionnaire was sent to all clinics in Great Britain. We asked which methods their clinic routinely used for diagnosis: (a) clinical appearance only (b) culture (c) microscopy (d) "other". If microscopy was used, respondents were asked to describe how samples were taken and examined.

\section{Results}

Of the 450 sub-preputial and penile glans swabs obtained from 1991 to 1993,158 (35\%) were culture positive for candida. Microscopy was performed on specimens from 447 patients. Forty-nine men were randomly selected to have material collected for microscopy by the "adhesive tape" method in addition to the "plain slide" method. The results of microscopy compared with culture are shown in table 1. Microscopy using the "plain slide" method of material collection had a sensitivity of $12 \%$ compared to $65 \%$ for the "adhesive tape" method of sampling (chi square, $\mathrm{p}<0.0001)$. The specificities of both methods were $95 \%$ and $81 \%$ respectively $\left(\chi^{2}\right.$ $p>0 \cdot 5)$. The positive predictive value for microscopy using the "plain slide" method of material collection was $50 \%$ compared with $75 \%$ for the "adhesive tape" method. The respective negative predictive values were $71 \%$ and $72 \%$.

The degree of discomfort produced by collecting material with adhesive tape compared with swabbing for candida culture is shown in table 2 .

Questionnaires were sent to 250 GUM clinics in Great Britain and replies obtained from $150(60 \%)$. Nineteen (13\%) routinely diagnosed candidal balano-posthitis by clinical appearance only and did not perform microscopy or culture. A further 51 (34\%) stated they sometimes relied solely on clinical appearance for diagnosis. Culture was used by

Table 2 Degree of discomfort produced by swabbing for culture and sampling for microscopy by adhesive tape

\begin{tabular}{lcc}
\hline & $\begin{array}{c}\text { Adhesive tape } \\
n(\%)\end{array}$ & $\begin{array}{l}\text { Swab } \\
n(\%)\end{array}$ \\
\hline $\begin{array}{l}\text { Degree of discomfort } \\
\text { on scale of 1-5 }\end{array}$ & \\
1 & $28(58 \%)$ & $6(18 \%)$ \\
2 & $8(16 \%)$ & $5(15 \%)$ \\
3 & $7(14 \%)$ & $8(23 \%)$ \\
4 & $3(6 \%)$ & $8(23 \%)$ \\
5 & $3(6 \%)$ & $7(21 \%)$ \\
Total & 49 & 34 \\
\hline I &
\end{tabular}

$117(78 \%)$ and microscopy by 104 (69\%). Eighty-eight clinics provided information on how the sample was taken for microscopy and examined. Sixty-one (69\%) used a dry cottonwool tipped swab for obtaining material, 11 (13\%) a moist swab, $8(9 \%)$ a dry loop, 5 (6\%) skin scrapings and $3(3 \%)$ a dry slide pressed against the penis. Considering the methods used for microscopy, 71 (81\%) used the Gram stain, $14(16 \%)$ a wet preparation with normal saline, $2(2 \%)$ a wet preparation with potassium hydroxide and $1(1 \%)$ an acridine orange stain.

\section{Discussion}

Balano-posthitis is a common condition. In one London GUM clinic, balanitis was diagnosed in $11 \%$ of men attending over a three month period. ${ }^{8}$ There is little information on the prevalence of candida as a cause of balanoposthitis. Although the diagnosis is often considered, many cases of presumed candidal infection show only histological features of a non-specific dermatitis. ${ }^{9}$ We identified candida in just over one third of men presenting to our department with balano-posthitis over a three year period. This is in contrast to studies of men with recurrent or persistent balanitis where Candida albicans has been reported in only $2 \%$ to $8 \%$ of cases. ${ }^{810}$

Following discussion with colleagues we were aware that candidal balano-posthitis is often diagnosed on clinical appearance alone. Although almost one half of the clinics responding to our questionnaire stated they would often rely solely on clinical appearances for diagnosis, only $13 \%$ did not perform microscopy or culture. We would suspect a high level of diagnostic accuracy when cases with classical clinical appearances are assessed by experienced clinicians; however, we have no data to support this view. Although microscopy is used by over two-thirds of clinics, the sensitivity may vary with the method of sampling. The sensitivity of microscopy was only $12 \%$ when material was collected by pressing a microscope slide against the penis. However, the use of an adhesive-tape method of sampling improved sensitivity to $65 \%$. This was well tolerated by patients and should be compared with the cotton-wool tipped swab method of sampling, which is currently used by $82 \%$ of the clinics performing microscopy. A number of false positive diagnoses were made by microscopy. Some of these were due to the presence of material which had a similar Gram stained appearance to hyphae or spores and which we suspect was debris from the subpreputial space and epithelial cell fragments. Small filaments of, possibly, material from underwear were also seen in a number of cases and had been misdiagnosed as hyphal strands. The presence of dead fungi may also have contributed to some of the false positive diagnoses on microscopy.

In summary, we have found candida to be an important cause of balano-posthitis amongst male GUM clinic attenders. The currently used diagnostic methods are worthy of 
further comparative study and would prove a useful topic for clinical audit.

We thank all those who kindly completed and returned our questionnaire. In addition, we thank $\mathrm{Dr} S \mathrm{O}$ Roberts for his questionnaire. In addition, we thank Dr S O Roberts for his from the Department of Microbiology, Addenbrooke's Hospital for their assistance and helpful comments.

1 Engman MF. A peculiar fungus infection of the skin (soorpilze?) Arch Dermatol Syphilol 1920;1:730.

2 Waugh MA, Evans ESV, Nayyar KC, Fong R. Clotrimazole in the treatment of candidal balanitis in men. $\mathrm{Br} \mathcal{F}$ Venereal Dis 1978;54:184-6.

3 Eddie DAS. The laboratory diagnosis of vaginal infections caused by Trichomonas and Candida (Monilia) species. 7 Med Microbiol 1968;1:153-9.

4 Bergman JJ, Berg AO, Schneeweiss R, Heidrich FE. Clinical comparison of microscopic and culture tech- niques in the diagnosis of candida vaginitis. 7 Fam Pract 1984;18:549.

5 Oates JK, Selwyn S, Breach MR. Polyester sponge swabs to facilitate examination for genital infection in women. $\mathrm{Br} f$ Venereal Dis 1971;47:289-92.

6 Feinberg JG, Whttington JM. A culture medium for Trichomonas vaginalis and species of candida. $f \mathrm{Clin}$ Pathol 1957;10:327-9.

7 Stenton P. The isolation of Trichomonas vaginalis. $7 \mathrm{Med}$ Lab Technol 1957;14:228-30.

8 Birley HDL, Walker MM, Luzzi GA, et al. Clinical features and management of recurrent balanitis: atopy and genital washing. Geni

9 Hillman RJ, Walker MM, Harris JRW, Taylor-Robinson D. Penile dermatoses: a clinical and histopathological study. Genitourin Med 1992;68:166-9.

10 Veller Fornasa C, Calabro A, Miglietta A, Tarantello M, Biasinutto C, Peserico A. Mild balanoposthitis. Genitourin Med 1994;70:345-6. 